\title{
The Fokas Method for the Broer-Kaup System on the Half-Line
}

\section{Guenbo Hwang ${ }^{1}$}

Received: 14 September 2021 / Accepted: 17 January 2022 / Published online: 21 February 2022

(c) The Author(s) 2022

\begin{abstract}
We analyze the Broer-Kaup system posed on the half-line by using the unified transform method, also known as the Fokas method. We derive the formal representation of the solution for the Broer-Kaup system in terms of the solution of the matrix Riemann-Hilbert problem formulated in the complex plane of the spectral parameter. The jump matrix is uniquely defined by the spectral functions that satisfy a certain relation, called the global relation involving the initial and boundary values. Furthermore, the spectral functions constructed from the initial values and the boundary values are investigated, plus their associated Riemann-Hilbert problems as the inverse problems.
\end{abstract}

Keywords Initial-boundary value problem · Broer-Kaup system · Fokas method · Inverse scattering transform

Mathematics Subject Classification $35 \mathrm{Q} 15 \cdot 37 \mathrm{~K} 10 \cdot 37 \mathrm{~K} 15$

\section{Introduction}

The Broer-Kaup system (BK)

$$
\left\{\begin{array}{l}
v_{t}=\frac{1}{2}\left(v^{2}\right)_{x}+w_{x}-\frac{1}{2} v_{x x}, \\
w_{t}=(w v)_{x}+\frac{1}{2} w_{x x}
\end{array}\right.
$$

arises as a model that describes the bi-directional propagation of long waves in shallow water [1,2]. Eq. (1) is a coupled integrable system induced from the Boussinesq equation $[1,3]$. Being integrable, the BK system has been widely examined such as the Lax pair and the inverse scattering transform [2, 4, 5], extended Painlevé

Guenbo Hwang

ghwang@daegu.ac.kr

1 Department of Mathematics and Institute of Natural Sciences, Daegu University, Gyeongsan, Gyeongbuk 38453, Korea 
expansion [6], perturbation theroy based on the inverse scattering transform [7], the Darboux transformation [8], a tri-Hamiltonian structure with an infinite number of conservation laws [9], the soliton solutions given by its trilinear form [10] and peaked solitary wave solutions $[11,12]$. In the context of the inverse scattering transform, the $2 \times 2$ matrix Lax pair has been analyzed in [4, 5]. It has been shown in [5] that there are two cases of eigenvalues; purely imaginary eigenvalues and complex conjugate pair of eigenvalues. The former case reduces to the elastic interaction of the solitons and the latter leads to the blow up and breather solutions.

The main purpose of the paper is to develop the inverse scattering transform for initial boundary value problems (IBVPs) of nonlinear integrable equations. More specifically, we are concerned with the IBVP for the BK system (1) formulated on the half-line

$$
\left\{(x, t) \in \mathbb{R}^{2} \mid 0 \leq x, \quad 0 \leq t \leq T\right\},
$$

for which the initial and boundary values satisfy

$$
\begin{aligned}
& v(x, 0)=v_{0}(x), \quad w(x, 0)=w_{0}(x), \\
& v(0, t)=f_{0}(t), \quad v_{x}(0, t)=f_{1}(t), \quad w(0, t)=g_{0}(t), \quad w_{x}(0, t)=g_{1}(t),
\end{aligned}
$$

where the functions $v_{0}(x)$ and $w_{0}(x)$ are assumed to be sufficiently smooth for $x>0$ and to decay fast as $x \rightarrow \infty$. We also assume that the functions $f_{j}(t)$ and $g_{j}(t)$ $(j=0,1)$ are sufficiently smooth for $t>0$ (and to decay rapidly as $t \rightarrow \infty$ if $T=\infty$ ). This BK system posed on the half-line can be analyzed by using the Fokas method, which is considered as a generalization of the inverse scattering transform for IBVPs of integrable systems. We remark that the Fokas method has been extensively applied for analyzing a large class of boundary value problems such as nonlinear integrable equations [13-16], linear evolution equations [17, 18], linear and nonlinear elliptic partial differential equations [19-21] and difference-differential equations [22, 23] (see also [24-26] for recent applications of the method to coupled integrable IBVPs).

The Fokas method has several efficient advantages for analyzing IBVPs of integrable systems. In particular, we note that (1) the spectral functions satisfy a certain algebraic relation called the global relation involving all initial and boundary values. This global relation allows one not only to establish the existence of the unique solution for IBVPs, but to characterize unknown boundary values that enter in the spectral functions [15]. For example, for the Dirichlet boundary value problem, the Neumann boundary value is unknown. In this case, it is necessary to characterize the unknown boundary value. This characterization can be done by analyzing the global relation, known as the generalized Dirichlet-to-Neumann map [27-29] (see also [30, 31] for further applications of the global relation). (2) The jump matrix of the Riemann-Hilbert problem has an explicit exponetial form of dependence on $x$ and $t$. Thus, it is possible to study long time asymptotics of the solution by using the Deift-Zhou method [32] or to study the small dispersion limit by using the DeiftVenakides-Zhou method [33]. Moreover, it also provides an efficient way to characterize the long-time asymptotics for unknown boundary data by using the perturbative approach [34-38]. It should be also remarked that the Fokas method is relatively 
simple, but effective in solving IBVPs for linear partial differential equations. The Fokas method presents an explicit integral representation of the solution for linear IBVPs, which also leads to efficient new numerical scheme, called a hybrid analytical-numerical method [39-41].

In this paper, assuming that the solution for the BK system exists, we show that it can be represented by the solution of the matrix Riemann-Hilbert problem formulated in the complex plane with the jump matrix given by the spectral functions constructed from the initial and boundary values. We also derive the global relation for the BK system that relates the spectral functions.

The outline of the paper is as follows. In Sect. 2, the Lax pair for the BK system is analyzed so as to define the appropriate eigenfunctions and the spectral functions, which are used to formulate the basic Riemann-Hilbert for the BK system posed on the half-line. In Sect. 3, we define the spectral functions from the initial values and the boundary values and we investigate their associated Riemann-Hilbert problems as the inverse problems. Finally, we end with some concluding remarks in Sect. 4.

\section{Spectral Analysis}

\subsection{Lax Pair and Eigenfunctions}

The BK system admits the following overdetermined linear systems, called the Lax pair $[4,5]$

$$
\begin{aligned}
& \Psi_{x}+i k\left[\sigma_{3}, \Psi\right]=U_{\mathrm{int}} \Psi, \\
& \Psi_{t}-k^{2}\left[\sigma_{3}, \Psi\right]=V_{\mathrm{int}} \Psi,
\end{aligned}
$$

where $k \in \mathbb{C}$ is a spectral parameter, $\Psi(x, t, k)$ is a $2 \times 2$ matrix-valued eigenfunction, $\sigma_{3}$ denotes the third Pauli matrix, namely, $\sigma_{3}=\operatorname{diag}(1,-1)$ and

$$
U_{\mathrm{int}}=\left(\begin{array}{cc}
\frac{v}{2} & 1 \\
-w & -\frac{v}{2}
\end{array}\right), \quad V_{\mathrm{int}}=\left(\begin{array}{cc}
\frac{r}{4} & i k+\frac{v}{2} \\
-i k w-\frac{q}{2} & -\frac{r}{4}
\end{array}\right)
$$

with $r=v^{2}-v_{x}$ and $q=w_{x}+w v$ for simplicity. Here, we assume that the real-valued functions $v$ and $w$ decay rapidly for all $t$ as $x \rightarrow \infty$. It is convenient to denote the matrix commutator simply by $\hat{\sigma}_{3}$; then $e^{\hat{\sigma}_{3}}$ can be easily computed as

$$
\hat{\sigma}_{3} A=\left[\sigma_{3}, A\right]=\sigma_{3} A-A \sigma_{3}, \quad e^{\hat{\sigma}_{3}}=e^{\sigma_{3}} A e^{-\sigma_{3}},
$$

where $A$ is a $2 \times 2$ matrix. Note that the Lax pair given in Eqs. (2) is not a standard form for defining special eigenfunctions that are well-controlled for large $k$. It should require to transform the original Lax pair into the form that $U_{\text {int }}$ and $V_{\text {int }}$ vanish as $x \rightarrow \infty$ and the leading order term for $k$ is off-diagonal. In this respect, we first define $\Psi(x, t, k)=D(k) \Psi_{\infty}(x, t, k)$, where 


$$
D(k)=\left(\begin{array}{cc}
1 & \frac{1}{2 i k} \\
0 & 1
\end{array}\right) .
$$

Then Eqs. (2) can be transformed into the form

$$
\begin{aligned}
& \Psi_{\infty x}+i k\left[\sigma_{3}, \Psi_{\infty}\right]=U_{\infty} \Psi_{\infty}, \\
& \Psi_{\infty t}-k^{2}\left[\sigma_{3}, \Psi_{\infty}\right]=V_{\infty} \Psi_{\infty},
\end{aligned}
$$

where

$$
U_{\infty}=\left(\begin{array}{cc}
\frac{v}{2}+\frac{w}{2 i k} & \frac{v}{2 i k}-\frac{w}{4 k^{2}} \\
-w & -\frac{v}{2}-\frac{w}{2 i k}
\end{array}\right), \quad V_{\infty}=\left(\begin{array}{cc}
\frac{1}{4}(r+2 w)+\frac{q}{4 i k} & \frac{v}{2}+\frac{1}{4 i k}(r+w)-\frac{q}{8 k^{2}} \\
-i k w-\frac{q}{2} & -\frac{1}{4}(r+2 w)-\frac{q}{4 i k}
\end{array}\right) .
$$

We expand $\Psi_{\infty}$ as

$$
\Psi_{\infty}(x, t, k)=\Psi_{\infty}^{(0)}(x, t)+\frac{\Psi_{\infty}^{(1)}(x, t)}{k}+O\left(1 / k^{2}\right), \quad(k \rightarrow \infty) .
$$

Substituting Eqs. (5) into (3a), we find

$$
\begin{gathered}
O(k): \quad i\left[\sigma_{3}, \Psi_{\infty}^{(0)}\right]=0, \\
O(1): \quad \Psi_{\infty x}^{(0)}+i\left[\sigma_{3}, \Psi_{\infty}^{(1)}\right]=\left(\begin{array}{cc}
\frac{v}{2} & 0 \\
-w & -\frac{v}{2}
\end{array}\right) \Psi_{\infty}^{(0)} .
\end{gathered}
$$

On the other hand, substituting Eq. (5) into the $t$-part of the Lax pair (3b), we obtain

$$
\begin{gathered}
O\left(k^{2}\right): \quad-\left[\sigma_{3}, \Psi_{\infty}^{(0)}\right]=0 \\
O(k): \quad-\left[\sigma_{3}, \Psi_{\infty}^{(1)}\right]=\left(\begin{array}{cc}
0 & 0 \\
-i w & 0
\end{array}\right) \Psi_{\infty}^{(0)}, \\
O(1): \quad \Psi_{\infty t}^{(0)}-\left[\sigma_{3}, \Psi_{\infty}^{(2)}\right]=\left(\begin{array}{cc}
\frac{1}{4}(r+2 w) & \frac{v}{2} \\
-\frac{q}{2} & -\frac{1}{4}(r+2 w)
\end{array}\right) \Psi_{\infty}^{(0)}+\left(\begin{array}{cc}
0 & 0 \\
-i w & 0
\end{array}\right) \Psi_{\infty}^{(1)} .
\end{gathered}
$$

Equations (6a) and (7a) are consistent and we find $\Psi_{\infty, 12}^{(0)}=\Psi_{\infty, 21}^{(0)}=0$. From Eqs. (6b) and (7c), it follows that

$$
\Psi_{\infty x}^{(0)}=\frac{v}{2} \sigma_{3} \Psi_{\infty}^{(0)}, \quad \Psi_{\infty t}^{(0)}=\frac{1}{4}(r+2 w) \sigma_{3} \Psi_{\infty}^{(0)}
$$

and hence we find 


$$
\Psi_{\infty}^{(0)}(x, t)=e^{\int_{\left(x_{0}, t_{0}\right)}^{(x, t)} \Delta \sigma_{3}}
$$

with the closed differential one-form defined by

$$
\Delta(x, t)=\Delta_{1} d x+\Delta_{2} d t=\frac{v}{2} d x+\frac{1}{4}(r+2 w) d t .
$$

For a simple calculation, we take $\left(x_{0}, t_{0}\right)=(0,0)$. The asymptotics for the eigenfunction $\Psi_{\infty}$ given in Eq. (5) suggests to introduce a new function $\mu(x, t, k)[16]$

$$
\Psi_{\infty}(x, t, k)=e^{\int_{(0,0)}^{(x, t)} \Delta \hat{\sigma}_{3}} \mu(x, t, k) \Psi_{\infty}^{(0)}(x, t) .
$$

Then we have

$$
\mu(x, t, k)=I+O(1 / k), \quad(k \rightarrow \infty)
$$

and Eqs. (3) can be written as

$$
d\left(e^{\left(i k x-k^{2} t\right) \hat{\sigma}_{3}} \mu(x, t, k)\right)=W(x, t, k),
$$

where the closed differential one-form is defined by

$$
W(x, t, k)=e^{\left(i k x-k^{2} t\right) \hat{\sigma}_{3}}[U d x+V d t] \mu(x, t, k)
$$

with

$$
\begin{gathered}
U(x, t, k)=\left(\begin{array}{cc}
\frac{w}{2 i k} & \left(\frac{v}{2 i k}-\frac{w}{4 k^{2}}\right) e^{-2 \int_{(0,0)}^{(x, t)} \Delta} \\
-w e^{2 \int_{(0,0)}^{(x, t)} \Delta} & -\frac{w}{2 i k}
\end{array}\right), \\
V(x, t, k)=\left(\begin{array}{c}
\frac{q}{4 i k} \\
\left(-i k w-\frac{q}{2}\right) e^{2 \int_{(0,0)}^{(x, t)} \Delta}
\end{array} \quad \begin{array}{c}
\left(\frac{v}{2}+\frac{1}{4 i k}(r+w)-\frac{q}{8 k^{2}}\right) e^{-2 \int_{(0,0)}^{(x, t)} \Delta} \\
-\frac{q}{4 i k}
\end{array}\right) .
\end{gathered}
$$

We also note that Eq. (12) is equivalent to the following modified Lax pair

$$
\begin{aligned}
& \mu_{x}+i k\left[\sigma_{3}, \mu\right]=U \mu, \\
& \mu_{t}-k^{2}\left[\sigma_{3}, \mu\right]=V \mu .
\end{aligned}
$$

It should be now remarked that $U, V \rightarrow 0$ as $x \rightarrow \infty$ and the leading order term for $k$ is off-diagonal. As a result, we define the Jost eigenfunction as the simultaneous solution for the both parts of the Lax pair (15)

$$
\mu_{j}(x, t, k)=I+\int_{\left(x_{j}, t_{j}\right)}^{(x, t)} e^{-\left(i k x-k^{2} t\right) \hat{\sigma}_{3}} W_{j}(\xi, \tau, k),
$$


where $(x, t),\left(x_{j}, t_{j}\right) \in\{0<x<\infty, 0<t<T\}$ and $W_{j}$ is the differential one-form defined by Eq. (13) with $\mu_{j}$. Note that since the one-form $W$ is exact, the integration in Eq. (16) is path-independent. Hence, we choose three distinct normalization points (cf. Fig. 1)

$$
\left(x_{1}, t_{1}\right)=(0,0), \quad\left(x_{2}, t_{2}\right)=(\infty, t), \quad\left(x_{3}, t_{3}\right)=(0, T) .
$$

More precisely, we define the Jost eigenfunctions that solve the following integral equations

$$
\begin{gathered}
\mu_{1}(x, t, k)=I+\int_{0}^{x} e^{-i k(x-\xi) \hat{\sigma}_{3}} U \mu_{1}(\xi, t, k) d \xi+e^{-i k x \hat{\sigma}_{3}} \int_{0}^{t} e^{k^{2}(t-\tau) \hat{\sigma}_{3}} V_{0}(\tau, k) \mu_{1}(0, \tau, k) d \tau, \\
\mu_{2}(x, t, k)=I-\int_{x}^{\infty} e^{-i k(x-\xi) \hat{\sigma}_{3}} U \mu_{2}(\xi, t, k) d \xi \\
\mu_{3}(x, t, k)=I+\int_{0}^{x} e^{-i k(x-\xi) \hat{\sigma}_{3}} U \mu_{3}(\xi, t, k) d \xi-e^{-i k x \hat{\sigma}_{3}} \int_{t}^{T} e^{k^{2}(t-\tau) \hat{\sigma}_{3}} V_{0}(\tau, k) \mu_{3}(0, \tau, k) d \tau,
\end{gathered}
$$

where $V_{0}(t, k)=V(0, t, k)$. Since $v$ and $w$ are real-valued, the potential functions $U$ and $V$ have the symmetry: $\overline{U(x, t,-\bar{k})}=U(x, t, k)$ and $\overline{V(x, t,-\bar{k})}=V(x, t, k)$, where the overline denotes the complex conjugation. Thus the eigenfunction $\mu$ has the symmetry $\overline{\mu(x, t,-\bar{k})}=\mu(x, t, k)$. Note that the off-diagonal components of the matrixvalued eigenfunction $\mu$ involve the explicit exponential terms. Thus, we partition the complex plane into the domains $D_{j}(j=1, \ldots, 4)$ defined as (see Fig. 2)

$$
\begin{aligned}
& D_{1}=\left\{k \in \mathbb{C} \mid \operatorname{Im} k>0 \text { and } \operatorname{Re} k^{2}>0\right\}=\{k \in \mathbb{C} \mid 0<\arg k<\pi / 4 \text { or } 3 \pi / 4<\arg k<\pi\}, \\
& D_{2}=\left\{k \in \mathbb{C} \mid \operatorname{Im} k<0 \text { and } \operatorname{Re} k^{2}>0\right\}=\{k \in \mathbb{C} \mid-\pi / 4<\arg k<0 \text { or } \pi<\arg k<5 \pi / 4\}, \\
& D_{3}=\left\{k \in \mathbb{C} \mid \operatorname{Im} k>0 \text { and } \operatorname{Re} k^{2}<0\right\}=\{k \in \mathbb{C} \mid \pi / 4<\arg k<3 \pi / 4\}, \\
& D_{4}=\left\{k \in \mathbb{C} \mid \operatorname{Im} k<0, \operatorname{Re} k^{2}<0\right\}=\{k \in \mathbb{C} \mid 5 \pi / 4<\arg k<7 \pi / 4\} .
\end{aligned}
$$

We denote $\mu^{(1)}$ and $\mu^{(2)}$ the columns of $2 \times 2$ matrix $\mu(x, t, k)=\left(\mu^{(1)}, \mu^{(2)}\right)$. We can determine regions, where the eigenfunctions are analytic and bounded as follows

- $\mu_{1}^{(1)}(x, t, k)$ is analytic for $k \in D_{1}$, and bounded for $k \in \bar{D}_{1}^{[\emptyset]} \cdot \mu_{1}^{(2)}(x, t, k)$ is analytic for $k \in D_{4}$, and bounded for $k \in \bar{D}_{4}^{[\emptyset]}$, where $\bar{D}$ is the closure of $D$ and $D^{[\emptyset]}=D \backslash\{0\}$.

Fig. 1 The eigenfunctions $\mu_{1}, \mu_{2}$ and $\mu_{3}$ for the Lax pair (15)

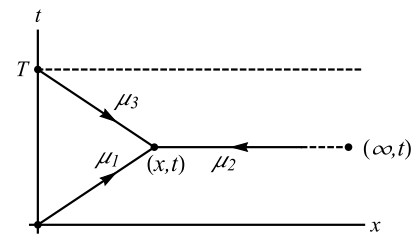



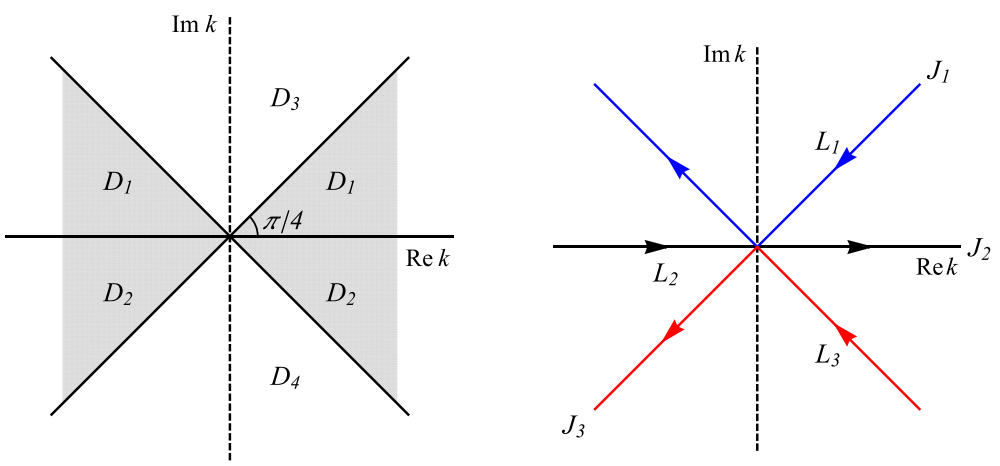

Fig. 2 (Left) The regions $D_{1}, \ldots, D_{4}$. The shaded is the region, where Re $k^{2}>0$. (Right) The oriented contours $L_{1}, L_{2}$ and $L_{3}$ that define the Riemann-Hilbert problem (see text for details)

- $\mu_{2}^{(1)}(x, t, k)$ is analytic for $k \in D_{2} \cup D_{4}$, and bounded for $k \in\left(\bar{D}_{2} \cup \bar{D}_{4}\right)^{[\emptyset]}$. $\mu_{2}^{(2)}(x, t, k)$ is analytic for $k \in D_{1} \cup D_{3}$, and bounded for $k \in\left(\bar{D}_{1} \cup \bar{D}_{3}\right)^{[\emptyset]}$.

- $\mu_{3}^{(1)}(x, t, k)$ is analytic for $k \in D_{3}$, and bounded for $k \in \bar{D}_{3}^{[\emptyset]} \cdot \mu_{3}^{(2)}(x, t, k)$ is analytic for $k \in D_{2}$, and bounded for $k \in \bar{D}_{2}^{[\emptyset]}$.

Moreover, the asymptotic behavior for the eigenfunction as $k \rightarrow \infty$ leads to the reconstruction formula for the solution of the BK system. As shown in Appendix, expanding

$$
\mu(x, t, k)=I+\frac{\chi^{(1)}(x, t)}{k}+\frac{\chi^{(2)}(x, t)}{k^{2}}+\cdots, \quad(k \rightarrow \infty),
$$

we find the asymptotic behavior of the eigenfunction $\mu$ :

$$
\mu(x, t, k)=I+\frac{1}{2 i k}\left(\begin{array}{cc}
\int_{(0,0)}^{(x, t)} \Omega & 0 \\
w e^{2 \int_{(0,0)}^{(x, t)} \Delta} & -\int_{(0,0)}^{(x, t)} \Omega
\end{array}\right)+O\left(1 / k^{2}\right), \quad(k \rightarrow \infty)
$$

with

$$
\chi_{12}^{(2)}(x, t)=-\frac{v}{4} e^{-2 \int_{(0,0)}^{(x, t)} \Delta},
$$

where the closed differential one-form $\Delta$ is given in eq. (10) and $\Omega$ is defined by

$$
\Omega(x, t)=w d x+\frac{1}{2}(q+w v) d t=\Omega_{1} d x+\Omega_{2} d t .
$$

We then have the reconstruction formula for the solution

$$
v e^{-2 \int_{(0,0)}^{(x, t)} \Delta}=-4 \lim _{k \rightarrow \infty} k^{2} \mu_{12}(x, t, k), \quad w e^{2 \int_{(0,0)}^{(x, t)} \Delta}=2 i \lim _{k \rightarrow \infty} k \mu_{21}(x, t, k) .
$$

We note that 


$$
\left(e^{-2 \int_{(0,0)}^{(x, t)} \Delta}\right)_{x}=-v e^{-2 \int_{(0,0)}^{(x, t)} \Delta}
$$

which implies that

$$
e^{-2 \int_{(0,0)}^{(x, t)} \Delta}=e^{-\int_{0}^{\infty} v_{0}(\xi) d \xi}-4 \int_{x}^{\infty} \chi_{12}^{(2)}(\xi, t) d \xi .
$$

Thus the inverse problem can be solved in the following steps: (1) Use any of the eigenfunctions $\mu_{j}(j=1,2,3)$ to find $m_{1}$ and $m_{2}$

$$
m_{1}(x, t)=\lim _{k \rightarrow \infty}\left(k \mu_{j}(x, t, k)\right)_{21}, \quad m_{2}(x, t)=\lim _{k \rightarrow \infty}\left(k^{2} \mu_{j}(x, t, k)\right)_{12} .
$$

(2) Determine $e^{-2 \int_{(0,0)}^{(x, t)} \Delta}$ given in Eq. (25). (3) The solution for the BK system on the half-line can be determined from $m_{1}$ and $m_{2}$ by

$$
v(x, t)=-4 m_{2}(x, t) e^{2 \int_{(0,0)}^{(x, t)} \Delta}, \quad w(x, t)=2 i m_{1}(x, t) e^{-2 \int_{(0,0)}^{(x, t)} \Delta} .
$$

Note that the matrix eigenfunctions are fundamental solutions of the Lax pair. Thus, they are related by the so-called scattering matrices $s(k)$ and $S(k)$, also known as the spectral matrices,

$$
\begin{aligned}
& \mu_{2}(x, t, k)=\mu_{1}(x, t, k) e^{-\left(i k x-k^{2} t\right) \hat{\sigma}_{3}} s(k), \\
& \mu_{3}(x, t, k)=\mu_{1}(x, t, k) e^{-\left(i k x-k^{2} t\right) \hat{\sigma}_{3}} S(k) .
\end{aligned}
$$

The spectral functions can be represented in terms of the eigenfunctions. Indeed, since $\mu_{1}(0,0, k)=I$, eq. (26a) yields

$$
s(k)=\mu_{2}(0,0, k) .
$$

Thus, the spectral matrix $s(k)$ can be expressed by

$$
s(k)=I-\int_{0}^{\infty} e^{i k x \hat{\sigma}_{3}} U \mu_{2}(x, 0, k) d x .
$$

On the other hand, noting that $\mu_{3}(0, T, k)=I$, Eq. (26b) implies that

$$
S(k)=\mu_{3}(0,0, k)=\left(e^{-k^{2} T \hat{\sigma}_{3}} \mu_{1}(0, T, k)\right)^{-1} .
$$

Thus, the spectral matrix $S(k)$ can be expressed in terms of the eigenfunction $\mu_{1}$

$$
S^{-1}(k)=I+\int_{0}^{T} e^{-k^{2} t \hat{\sigma}_{3}} V_{0}(t, k) \mu_{1}(0, t, k) d t .
$$

Hereafter, we write the spectral matrices as 


$$
s(k)=\left(\begin{array}{ll}
a_{11}(k) & a_{12}(k) \\
a_{21}(k) & a_{22}(k)
\end{array}\right), \quad S(k)=\left(\begin{array}{cc}
A_{11}(k) & A_{12}(k) \\
A_{21}(k) & A_{22}(k)
\end{array}\right) .
$$

Note that $s(k)$ and $S(k)$ enjoy the same symmetry as the eigenfunction, namely, $\overline{s(-\bar{k})}=s(k)$ and $\overline{S(-\bar{k})}=S(k)$. Similarly, we can determine the regions, where the spectral functions $s(k)$ and $S(k)$ are analytic and bounded:

- $s^{(1)}(k)$ is analytic for $k \in D_{2} \cup D_{4}$, and bounded for $k \in\left(\bar{D}_{2} \cup \bar{D}_{4}\right)^{[\emptyset]} . s^{(2)}(k)$ is analytic for $k \in D_{1} \cup D_{3}$, and bounded for $k \in\left(\bar{D}_{1} \cup \bar{D}_{3}\right)^{[\emptyset]}$.

- $\operatorname{det} s(k)=1$ and $s(k)=I+O(1 / k)$ as $k \rightarrow \infty$ in the respective domains of boundedness of the columns.

- $S(k)$ is analytic for $k \in \mathbb{C}^{[\emptyset]}$ if $T<\infty ; S^{(1)}(k)$ is bounded for $k \in\left(\bar{D}_{3} \cup \bar{D}_{4}\right)^{[\emptyset]}$, and $S^{(2)}(k)$ is bounded for $k \in\left(\bar{D}_{1} \cup \bar{D}_{2}\right)^{[\emptyset]}$. If $T=\infty$, the spectral functions $S^{(1)}(k)$ and $S^{(2)}(k)$ are defined for $k \in\left(\bar{D}_{3} \cup \bar{D}_{4}\right)^{[\emptyset]}$ and $k \in\left(\bar{D}_{1} \cup \bar{D}_{2}\right)^{[\emptyset]}$, respectively.

- $\operatorname{det} S(k)=1$ and $S(k)=I+O(1 / k)$ as $k \rightarrow \infty$ in the respective domains of boundedness of the columns.

Furthermore, eqs. (27) and (28) imply the relation

$$
\mu_{3}(x, t, k)=\mu_{2}(x, t, k) e^{-\left(i k x-k^{2} t\right) \hat{\sigma}_{3}} s^{-1}(k) S(k),
$$

where the first column is defined for $k \in D_{2} \cup D_{4}$ and the second column holds for $k \in D_{1} \cup D_{3}$, depending on the eigenfunction $\mu_{2}$. Evaluating the above equation at $(x, t)=(0, T)$, the spectral functions satisfy the following relation, known as the global relation

$$
S^{-1}(k) s(k)=e^{-k^{2} T \hat{\sigma}_{3}} \mu_{2}(0, T, k), \quad k \in\left(D_{2} \cup D_{4}, D_{1} \cup D_{3}\right) .
$$

\subsection{Riemann-Hilbert Problem}

We will formulate the matrix Riemann-Hilbert problem for the BK system. For later reference, we introduce the quantities

$$
\begin{gathered}
\theta(x, t, k)=-i k x+k^{2} t, \quad d_{1}(k)=a_{12}(k) A_{21}(k)-A_{11}(k) a_{22}(k), \\
d_{2}(k)=a_{11}(k) A_{22}(k)-A_{12}(k) a_{21}(k), \quad d_{3}(k)=a_{12}(k) A_{22}(k)-A_{12}(k) a_{22}(k), \\
\Gamma_{1}(k)=\frac{A_{21}(k)}{a_{22}(k) d_{1}(k)}, \quad \Gamma_{2}(k)=\frac{A_{12}(k)}{a_{11}(k) d_{2}(k)}, \quad \Gamma_{3}(k)=\frac{A_{22}(k)}{a_{22}(k) d_{2}(k)}
\end{gathered}
$$

After some tedious but straightforward algebra, from Eqs. (26a) and (26b), we can define the following Riemann-Hilbert problem

$$
M_{-}(x, t, k)=M_{+}(x, t, k) J(x, t, k), \quad k \in L,
$$

where the sectionally meromorphic functions $M_{ \pm}$are defined by 


$$
\begin{aligned}
& M_{+}(x, t, k)=\left\{\begin{array}{l}
\left(\frac{\mu_{1}^{(1)}(x, t, k)}{a_{22}(k)}, \mu_{2}^{(2)}(x, t, k)\right), k \in D_{1} \\
\left(\mu_{2}^{(1)}(x, t, k), \frac{\mu_{1}^{(2)}(x, t, k)}{a_{11}(k)}\right), k \in D_{4}
\end{array}\right. \\
& M_{-}(x, t, k)=\left\{\begin{array}{l}
\left(\mu_{2}^{(1)}(x, t, k), \frac{\mu_{3}^{(2)}(x, t, k)}{d_{2}(k)}\right), k \in D_{2} \\
\left(\frac{\mu_{3}^{(1)}(x, t, k)}{d_{1}(k)}, \mu_{2}^{(2)}(x, t, k)\right), \\
k \in D_{3}
\end{array}\right.
\end{aligned}
$$

and the jump matrices are given by

$$
\begin{aligned}
& J_{1}=\left(\begin{array}{cc}
-1 & 0 \\
e^{-2 \theta(x, t, k)} \Gamma_{1}(k) & 1
\end{array}\right), \quad k \in L_{1}, \quad J_{2}=\left(\begin{array}{cc}
1 & -\mathrm{e}^{2 \theta(x, t, k)} \frac{d_{3}(k)}{d_{2}(k)} \\
e^{-2 \theta(x, t, k)} \frac{a_{21}(k)}{a_{22}(k)} & \Gamma_{3}(k)
\end{array}\right), \quad k \in L_{2}, \\
& J_{3}=\left(\begin{array}{cc}
1 & e^{2 \theta(x, t, k)} \Gamma_{2}(k) \\
0 & 1
\end{array}\right), \quad k \in L_{3}
\end{aligned}
$$

with the oriented contour $L$ (see Fig. 2)

$$
L_{1}=\bar{D}_{1} \cap \bar{D}_{3}, \quad L_{2}=\bar{D}_{1} \cap \bar{D}_{2}, \quad L_{3}=\bar{D}_{2} \cap \bar{D}_{4},
$$

where the orientation of $L$ is taken so that the regions denoted by + are on the left of the positive direction of $L$. Note that $\operatorname{det} M_{ \pm}=1$ and $M_{ \pm}(x, t, k)=I+O(1 / k)$ as $k \rightarrow \infty$ in the respective domains of boundedness of their columns.

The solution for the BK system can be found from the solution of the RiemannHilbert problem. In this respect, we expand the solution $M(x, t, k)$ of the RiemannHilbert problem as

$$
M(x, t, k)=I+\frac{M_{1}(x, t)}{k}+\frac{M_{2}(x, t)}{k^{2}}+O\left(1 / k^{3}\right), \quad(k \rightarrow \infty) .
$$

Letting $M_{+}-M_{-}=M_{+} \tilde{J}$, where $\tilde{J}=I-J$, the Riemann-Hilbert problem can be solved by the Cauchy type of integral equation

$$
M(x, t, k)=I+\frac{1}{2 i \pi} \int_{L} \frac{M_{+} \tilde{J}(x, t, l)}{l-k} d l .
$$

Then

$$
M_{1}(x, t)=-\frac{1}{2 i \pi} \int_{L} M_{+} \tilde{J}(x, t, l) d l, \quad M_{2}(x, t)=-\frac{1}{2 i \pi} \int_{L} M_{+} \tilde{J}(x, t, l) l d l
$$

and we can find the reconstruction formula for the solution of the BK system in terms of the solution of the Riemann-Hilbert problem

$$
v e^{-2 \int_{(0,0)}^{(x, t)} \Delta}=\frac{2}{i \pi} \int_{L}\left(M_{+} \tilde{J}(x, t, k)\right)_{12} k d k, \quad w e^{2 \int_{(0,0)}^{(x, t)} \Delta}=-\frac{1}{\pi} \int_{L}\left(M_{+} \tilde{J}(x, t, k)\right)_{21} d k .
$$

We note that the function $M(x, t, k)$ is sectionally meromorphic. The possible poles occur at the zeros of $a_{11}(k), a_{22}(k), d_{1}(k)$ and $d_{2}(k)$. Also, note that $\overline{s(-\bar{k})}=s(k)$ and 
$\overline{S(-\bar{k})}=S(k)$. We assume that there are a finite number of simple zeros. More precisely, we assume that

(i) $a_{11}(k)$ has a finite number of simple zeros in $D_{4}$. There are two types of zeros: $k_{j} \neq-\bar{k}_{j}$ and $k_{j}=-\bar{k}_{j}$ (purely imaginary). We assume that $a_{11}(k)$ has $2 n_{1}$ simple zeros at $k_{j}$ and $-\overline{k_{j}}$ with $k_{j} \neq-\overline{k_{j}}$ for $j=1,2, \ldots, n_{1}$ and assume that $a_{11}(k)$ has $n_{1}^{\prime}$ simple zeros at $k=i b_{j}\left(b_{j}<0\right)$ for $j=1,2, \ldots, n_{1}^{\prime}$.

(ii) $a_{22}(k)$ has $2 n_{2}$ simple zeros in $D_{1}$. We label such zeros $\tilde{k}_{j}$ and $-\tilde{k}_{j}$ for $j=1,2, \ldots, n_{2}$.

(iii) $d_{1}(k)$ has a finite number of simple zeros in $D_{3}$. We assume that $d_{1}(k)$ has $2 N_{1}$ simple zeros at $z_{j}$ and $-\overline{z_{j}}$ with $z_{j} \neq-\overline{z_{j}}$ for $j=1,2, \ldots, N_{1}$ and assume that $d_{1}(k)$ has $N_{1}^{\prime}$ simple zeros at $k=i c_{j}\left(c_{j}>0\right)$ for $j=1,2, \ldots, N_{1}^{\prime}$.

(iv) $d_{2}(k)$ has $2 N_{2}$ simple zeros in $D_{2}$. We label such zeros $\tilde{z}_{j}$ and $-\bar{z}_{j}$ for $j=1,2, \ldots, N_{2}$.

We then find the residue conditions

$$
\begin{gathered}
\operatorname{Res}_{k=k_{j}} M_{+}^{(2)}(x, t, k)=e^{2 \theta\left(x, t, k_{j}\right)} \frac{M_{+}^{(1)}\left(x, t, k_{j}\right)}{a_{21}\left(k_{j}\right) \dot{a}_{11}\left(k_{j}\right)}, \\
\operatorname{Res}_{k=\tilde{k}_{j}} M_{+}^{(1)}(x, t, k)=e^{-2 \theta\left(x, t, \tilde{k}_{j}\right)} \frac{M_{+}^{(2)}\left(x, t, \tilde{k}_{j}\right)}{a_{12}\left(\tilde{k}_{j}\right) \dot{a}_{22}\left(\tilde{k}_{j}\right)}, \\
\operatorname{Res}_{k=z_{j}} M_{-}^{(1)}(x, t, k)=e^{-2 \theta\left(x, t, z_{j}\right)} \frac{A_{21}\left(z_{j}\right) M_{-}^{(2)}\left(x, t, z_{j}\right)}{a_{22}\left(z_{j}\right) \dot{d}_{1}\left(z_{j}\right)}, \\
\operatorname{Res}_{k=\tilde{z}_{j}} M_{-}^{(2)}(x, t, k)=e^{2 \theta\left(x, t, \tilde{z}_{j}\right)} \frac{A_{22}\left(\tilde{z}_{j}\right) M_{-}^{(1)}\left(x, t, \tilde{z}_{j}\right)}{a_{21}\left(\tilde{z}_{j}\right) \dot{d}_{2}\left(\tilde{z}_{j}\right)},
\end{gathered}
$$

where the overdot denotes differentiation with respect to $k$. Indeed, recalling $\mu_{2}^{(1)}=a_{11} \mu_{1}^{(1)}+e^{-2 \theta} a_{21} \mu_{1}^{(2)}$, where we have suppressed the $x, t$ and $k$ dependence for simplicity, we can compute the residue

$$
\operatorname{Res}_{k=k_{j}} M_{+}^{(2)}(x, t, k)=\frac{\mu_{1}^{(2)}\left(x, t, k_{j}\right)}{\dot{a}_{11}\left(k_{j}\right)}=e^{2 \theta\left(x, t, k_{j}\right)} \frac{\mu_{2}^{(1)}\left(x, t, k_{j}\right)}{a_{21}\left(k_{j}\right) \dot{a}_{11}\left(k_{j}\right)},
$$

which is the first equation of eq. (35a). For eq. (35b), using $M_{-}=M_{+} J_{1}$ yields $a_{22} \mu_{3}^{(1)}=-d_{1} \mu_{2}^{(1)}+e^{-2 \theta} A_{21} \mu_{2}^{(2)}$. Thus, we find

$$
\operatorname{Res}_{k=z_{j}} M_{-}^{(1)}(x, t, k)=\frac{\mu_{3}^{(1)}\left(x, t, z_{j}\right)}{\dot{d}_{1}\left(z_{j}\right)}=e^{-2 \theta\left(x, t, z_{j}\right)} \frac{A_{21}\left(z_{j}\right) \mu_{2}^{(2)}\left(x, t, z_{j}\right)}{a_{22}\left(z_{j}\right) \dot{d}_{1}\left(z_{j}\right)},
$$

which is the first equation of Eq. (35b). Similarly, we can derive the second equations of Eqs. (35a) and (35b). We remark that for the purely imaginary zeros, the residue conditions given in Eqs. (35) are valid. 


\section{Spectral Functions}

Motivated by the analysis of Sect. 2, we define the spectral functions.

Definition 3.1 Given $v_{0}(x), w_{0}(x) \in \mathcal{S}\left(\mathbb{R}^{+}\right)$, we define the map

$$
\mathbb{S}:\left\{v_{0}(x), w_{0}(x)\right\} \rightarrow\left\{a_{i j}(k)\right\}, \quad(i, j=1,2)
$$

by $s(k)=\left(a_{i j}\right)=\mu_{2}(0, k)$, where

$$
\mu_{2}(x, k)=I-\int_{x}^{\infty} e^{-i k(x-\xi) \hat{\sigma}_{3}} U(\xi, 0, k) \mu_{2}(\xi, k) d \xi .
$$

Proposition 3.1 The spectral function $s(k)$ has the following properties:

(i) $s^{(1)}$ is analytic for $\operatorname{Im} k<0$ and bounded on $\operatorname{Im} k \leq 0$ except for $k=0 ; s^{(2)}$ is analytic $\operatorname{Im} k>0$ and bounded on $\operatorname{Im} k \geq 0$ except for $k=0$.

(ii) $s^{(1)}=\left(\begin{array}{l}1 \\ 0\end{array}\right)+O(1 / k)$ as $k \rightarrow \infty$ for $\operatorname{Im} k \leq 0 ; s^{(2)}=\left(\begin{array}{l}0 \\ 1\end{array}\right)+O(1 / k)$ as $k \rightarrow \infty$ for $\operatorname{Im} k \geq 0$.

(iii) $\underline{\operatorname{det} s(k)}=1$, that is, $a_{11}(k) a_{22}(k)-a_{12}(k) a_{21}(k)=1$ for $k \in \mathbb{R}^{[\emptyset]}$.

(iv) $s(-\bar{k})=s(k)$.

(v) The inverse map $\mathbb{S}^{-1}:\left\{a_{i j}(k)\right\} \rightarrow\left\{v_{0}(x), w_{0}(x)\right\}$ to the map $\mathbb{S}$ is defined by

$v_{0}(x) e^{-\int_{0}^{x} v_{0}(\xi) d \xi}=-4 M_{2}^{(x)}(x), \quad w_{0}(x) e^{\int_{0}^{x} v_{0}(\xi) d \xi}=2 i M_{1}^{(x)}(x)$,

where

$M_{1}^{(x)}(x)=\lim _{k \rightarrow \infty} k M_{21}^{(x)}(x, k), \quad M_{2}^{(x)}(x)=\lim _{k \rightarrow \infty} k^{2} M_{12}^{(x)}(x, k)$

and $M^{(x)}(x, k)$ is the unique solution of the following Riemann-Hilbert problem:

$$
M^{(x)}(x, k)=\left\{\begin{array}{l}
M_{+}^{(x)}(x, k), k \in \mathbb{C}^{+}, \\
M_{-}^{(x)}(x, k), k \in \mathbb{C}^{-}
\end{array}\right.
$$

is a meromorphic function for $k \in \mathbb{C} \backslash \mathbb{R}$, where $\mathbb{C}^{ \pm}$denote the upper/half plane of the complex plane, respectively.

- $M_{+}^{(x)}(x, k)-M_{-}^{(x)}(x, k)=M_{+}^{(x)}(x, k) J^{(x)}(x, k), \quad k \in \mathbb{R}$,

where $\mathbb{R}$ is oriented so that $k \in \mathbb{R}$ is increasing and the jump matrix $J^{(x)}$ is given by

$$
J^{(x)}(x, k)=\left(\begin{array}{cc}
\rho_{1}(k) \rho_{2}(k) & e^{-2 i k x} \rho_{2}(k) \\
-e^{2 i k x} \rho_{1}(k) & 0
\end{array}\right)
$$

with

$$
\rho_{1}(k)=\frac{a_{21}(k)}{a_{11}(k)}, \quad \rho_{2}(k)=\frac{a_{12}(k)}{a_{22}(k)} .
$$


- $\quad$ Assume that the first column of $M_{-}^{(x)}$ has $2 n_{3}$ simple zeros, labeled by $k_{j}$ and $-\overline{k_{j}}$ with $k_{j} \neq-\bar{k}_{j}\left(j=1,2, \ldots, n_{3}\right)$ and has $n_{3}^{\prime}$ simple zeros at $k=i b_{j}$ $\left(b_{j}<0\right.$ and $\left.j=1,2, \ldots, n_{3}^{\prime}\right)$. Furthermore, assume that the second column of $M_{+}^{(x)}$ has $2 n_{4}$ simple zeros, labeled by $\tilde{k}_{j}$ and $-\tilde{\tilde{k}}_{j}$ with $\tilde{k}_{j} \neq-\tilde{\tilde{k}}_{j}\left(j=1,2, \ldots, n_{4}\right)$ and has $n_{4}^{\prime}$ simple zeros at $k=i c_{j}\left(c_{j}>0\right.$ and $\left.j=1,2, \ldots, n_{4}^{\prime}\right)$. Then

$$
\operatorname{Res}_{k=k_{j}} M^{(x, 1)}(x, k)=\frac{e^{2 i k_{j} x} a_{21}\left(k_{j}\right)}{\dot{a}_{11}\left(k_{j}\right)} M^{(x, 2)}\left(x, k_{j}\right), \quad \operatorname{Res}_{k=\tilde{k}_{j}} M^{(x, 2)}(x, k)=\frac{e^{-2 i \tilde{k}_{j} x} a_{12}\left(\tilde{k}_{j}\right)}{\dot{a}_{22}\left(\tilde{k}_{j}\right)} M^{(x, 1)}\left(x, \tilde{k}_{j}\right),
$$

where $M^{(x, 1)}$ and $M^{(x, 2)}$ are the first column and the second column of the matrix $M^{(x)}$, respectively.

Proof (i)-(iv) can be proved from the discussion in Sect. 2.2. In order to prove (v), we define the eigenfunctions of the $x$-part of the Lax pair (15a) evaluated at $t=0$

$$
\begin{aligned}
& \mu_{1}(x, k)=I+\int_{0}^{x} e^{-i k(x-\xi) \hat{\sigma}_{3}} U(\xi, 0, k) \mu_{1}(\xi, k) d \xi \\
& \mu_{2}(x, k)=I-\int_{x}^{\infty} e^{-i k(x-\xi) \hat{\sigma}_{3}} U(\xi, 0, k) \mu_{2}(\xi, k) d \xi
\end{aligned}
$$

From Eq. (26a) evaluated at $t=0$, it follows that

$$
\mu_{2}(x, k)=\mu_{1}(x, k) e^{-i k x \hat{\sigma}_{3}} s(k) .
$$

Letting

$$
\begin{array}{ll}
M_{+}^{(x)}=\left(\mu_{1}^{(1)}(x, k), \frac{\mu_{2}^{(2)}(x, k)}{a_{22}(k)}\right), \quad \operatorname{Im} k>0, \\
M_{-}^{(x)}=\left(\frac{\mu_{2}^{(1)}(x, k)}{a_{11}(k)}, \mu_{1}^{(2)}(x, k),\right), \quad \operatorname{Im} k<0,
\end{array}
$$

Eq. (44) can be written as the Riemann-Hilbert problem defined by in Eq. (40) with the jump matrix given in Eq. (41). Moreover, Eq. (44) yields the residue conditions given by Eq. (42).

As shown in Sect. 2.1 (cf. Appendix), we have the asymptotic expansion for $M^{(x)}(x, k)$

$$
M^{(x)}(x, k)=I+\frac{1}{2 i k}\left(\begin{array}{cc}
\int_{0}^{x} w_{0}(\xi) d \xi & 0 \\
w_{0} e^{\int_{0}^{x} v_{0}(\xi) d \xi} & -\int_{0}^{x} w_{0}(\xi) d \xi
\end{array}\right)+O\left(1 / k^{2}\right), \quad(k \rightarrow \infty)
$$

with

$$
-\frac{v_{0}}{4} e^{-\int_{0}^{x} v_{0}(\xi) d \xi}=\lim _{k \rightarrow \infty} k^{2} M_{12}^{(x)}
$$

Thus, eq. (38) can be derived, where 


$$
e^{-\int_{0}^{x} v_{0}(\xi) d \xi}=4 \int_{0}^{x} M_{2}^{(x)}(\xi) d \xi+1
$$

Definition 3.2 Given smooth functions $f_{n}(t), g_{n}(t)(n=0,1)$, we define the map

$$
\mathbb{Q}:\left\{f_{n}(t), g_{n}(t)\right\} \rightarrow\left\{A_{i j}(k)\right\}, \quad(i, j=1,2)
$$

by $S(k)=\left(A_{i j}\right)=\mu_{3}(0, k)$, where

$$
\mu_{3}(t, k)=I-\int_{t}^{T} e^{k^{2}(t-\tau) \hat{\sigma}_{3}} V_{0}(\tau, k) \mu_{3}(\tau, k) d \tau .
$$

Proposition 3.2 The spectral function $S(k)$ has the following properties:

(i) $S^{(1)}$ is analytic for $k \in D_{3} \cup D_{4}$ and bounded for $k \in\left(\bar{D}_{3} \cup \bar{D}_{4}\right)^{[\emptyset]}$; whereas $S^{(2)}$ is analytic for $k \in D_{1} \cup D_{2}$ and bounded for $k \in\left(\bar{D}_{1} \cup \bar{D}_{2}\right)^{[\emptyset]}$.

(ii) $S^{(1)}=\left(\begin{array}{l}1 \\ 0\end{array}\right)+O(1 / k)$ as $k \rightarrow \infty$ for $k \in D_{3} \cup D_{4} ; S^{(2)}=\left(\begin{array}{l}0 \\ 1\end{array}\right)+O(1 / k)$ as $k \rightarrow \infty$ for $k \in D_{1} \cup D_{2}$.

(iii) $\operatorname{det} S(k)=1$, that is, $A_{11}(k) A_{22}(k)-A_{12}(k) A_{21}(k)=1$ for $k \in \mathbb{C}^{[\emptyset]}$ (if $T=\infty$, $\left.\underline{k^{2} \in} i \mathbb{R}^{[\emptyset]}\right)$.

(iv) $S(-\bar{k})=S(k)$.

(v) The inverse map $\mathbb{Q}^{-1}:\left\{A_{i j}(k)\right\} \rightarrow\left\{f_{n}(x), g_{n}(x)\right\}$ to the map $\mathbb{Q}$ is defined by

$$
\begin{aligned}
& f_{0} e^{-2 \int_{0}^{t} \Delta_{2}(\tau) d \tau}=-4\left(M_{2}^{(t)}(t)\right)_{12}, \quad g_{0} e^{2 \int_{0}^{t} \Delta_{2}(\tau) d \tau}=2 i\left(M_{1}^{(t)}(t)\right)_{21}, \\
& \int_{0}^{t} \Omega_{2}(\tau) d \tau=2 i\left(M_{1}^{(t)}(t)\right)_{11}, \\
& \quad\left(g_{1}+f_{0} g_{0}\right) e^{2 \int_{0}^{t} \Delta_{2}(\tau) d \tau}=4\left[\left(M_{1}^{(t)}(t)\right)_{11}\left(M_{1}^{(t)}(t)\right)_{21}-\left(M_{2}^{(t)}(t)\right)_{21}\right],
\end{aligned}
$$

where

$$
\Delta_{2}(t)=\frac{1}{4}\left(f_{0}^{2}(t)-f_{1}(t)+2 g_{0}(t)\right), \quad \Omega_{2}(t)=\frac{1}{2}\left(g_{1}(t)+2 g_{0}(t) f_{0}(t)\right),
$$

and the matrix functions $M_{1}^{(t)}(t)$ and $M_{2}^{(t)}(t)$ are determined by the asymptotic expansion

$$
M^{(t)}(t, k)=I+\frac{M_{1}^{(t)}(t)}{k}+\frac{M_{2}^{(t)}(t)}{k^{2}}+O\left(1 / k^{3}\right), \quad(k \rightarrow \infty),
$$

where $M^{(t)}(t, k)$ is the unique solution of the following Riemann-Hilbert problem: 


$$
M^{(t)}(t, k)=\left\{\begin{array}{l}
M_{+}^{(t)}(t, k), k \in D_{1} \cup D_{2}, \\
M_{-}^{(t)}(t, k), k \in D_{3} \cup D_{4}
\end{array}\right.
$$

is a meromorphic function for $k \in \mathbb{C} \backslash\left(L_{1} \cup L_{3}\right)$.

$$
\text { - } M_{+}^{(t)}(t, k)-M_{-}^{(t)}(t, k)=M_{+}^{(t)}(t, k) J^{(t)}(t, k), \quad k \in L_{1} \cup L_{3},
$$

where the orientation of $L_{1} \cup L_{3}$ is taken so that the regions denoted by + are on the left of the positive direction of $L_{1} \cup L_{3}$ and the jump matrix $J^{(t)}$ is given by

$$
J^{(t)}(t, k)=\left(\begin{array}{cc}
R_{1}(k) R_{2}(k) & e^{2 k t^{2}} R_{2}(k) \\
-e^{-2 k t^{2}} R_{1}(k) & 0
\end{array}\right)
$$

with

$$
R_{1}(k)=\frac{A_{21}(k)}{A_{11}(k)}, \quad R_{2}(k)=\frac{A_{12}(k)}{A_{22}(k)} .
$$

- Assume that the first column of $M_{-}^{(t)}$ has $2 N_{3}$ simple zeros, labeled by $\lambda_{j}$ and $-\bar{\lambda}_{j}\left(j=1,2, \ldots, N_{3}\right)$ and ass $\underline{\tilde{x}_{j}}\left(j\right.$ that the second column of $M_{+}^{(t)}$ has $2 N_{4}$ simple zeros, labeled by $\tilde{\lambda}_{j}$ and $-\tilde{\lambda}_{j}\left(j=1,2, \ldots, N_{4}\right)$. Then

$$
\begin{aligned}
& \operatorname{Res}_{k=\lambda_{j}} M^{(t, 1)}(t, k)=\frac{e^{-2 \lambda_{j}^{2} t} A_{21}\left(\lambda_{j}\right)}{\dot{A}_{11}\left(\lambda_{j}\right)} M^{(t, 2)}\left(t, \lambda_{j}\right), \\
& \operatorname{Res}_{k=\tilde{\lambda}_{j}} M^{(t, 2)}(t, k)=\frac{e^{2 i \tilde{\lambda}_{j}^{2} t} A_{12}\left(\tilde{\lambda}_{j}\right)}{\dot{A}_{22}\left(\tilde{\lambda}_{j}\right)} M^{(t, 1)}\left(t, \tilde{\lambda}_{j}\right),
\end{aligned}
$$

where $M^{(t, 1)}$ and $M^{(t, 2)}$ are the first column and the second column of the matrix $M^{(t)}$, respectively.

Proof It is enough to prove (v). Define the eigenfunctions of the $t$-part of the Lax pair (2b) evaluated at $x=0$

$$
\begin{aligned}
& \mu_{1}(t, k)=I+\int_{0}^{t} e^{k^{2}(t-\tau) \hat{\sigma}_{3}} V_{0}(\tau, k) \mu_{1}(\tau, k) d \tau, \\
& \mu_{3}(t, k)=I-\int_{t}^{T} e^{k^{2}(t-\tau) \hat{\sigma}_{3}} V_{0}(\tau, k) \mu_{3}(\tau, k) d \tau .
\end{aligned}
$$

From Eq. (26b) evaluated at $x=0$, it follows that

$$
\mu_{3}(t, k)=\mu_{1}(t, k) e^{k^{2} t \hat{\sigma}_{3}} S(k)
$$

\section{Letting}




$$
\begin{gathered}
M_{+}^{(t)}=\left(\mu_{1}^{(1)}(t, k), \frac{\mu_{3}^{(2)}(t, k)}{A_{22}(k)}\right), \quad k \in D_{1} \cup D_{2}, \\
M_{-}^{(t)}=\left(\frac{\mu_{3}^{(1)}(t, k)}{A_{11}(k)}, \mu_{1}^{(2)}(t, k),\right), \quad k \in D_{3} \cup D_{4} .
\end{gathered}
$$

Eq. (55) can be written as the Riemann-Hilbert problem defined by in Eq. (51) with the jump matrix given in Eq. (52). Moreover, Eq. (55) yields the residue conditions given by Eq. (53). Note that the asymptotic expansion for $M^{(t)}(t, k)$ becomes

$$
M^{(t)}(t, k)=I+\frac{1}{2 i k}\left(\begin{array}{cc}
\int_{0}^{t} \Omega_{2}(\tau) d \tau & 0 \\
g_{0} e^{2 \int_{0}^{t} \Delta_{2}(\tau) d \tau} & -\int_{0}^{t} \Omega_{2}(\tau) d \tau
\end{array}\right)+O\left(1 / k^{2}\right), \quad(k \rightarrow \infty)
$$

with

$$
\left(M_{2}^{(t)}(t)\right)_{12}=-\frac{f_{0}}{4} e^{-2 \int_{0}^{t} \Delta_{2}(\tau) d \tau}, \quad\left(M_{2}^{(t)}(t)\right)_{21}=-\frac{1}{4}\left(g_{1}+f_{0} g_{0}+g_{0} \int_{0}^{t} \Omega_{2}(\tau) d \tau\right) e^{2 \int_{0}^{t} \Delta_{2}(\tau) d \tau} .
$$

Thus, Eqs. (48) can be derived.

\section{Concluding Remarks}

In this work, we studied the IBVP for the BK system posed on the half-line by using the Fokas method. The Lax pair first should be transformed into a standard form so that we can define the well-controlled eigenfunctions for large spectral parameter $k$. We remark that the solution can be represented in terms of the solution of the matrix Riemann-Hilbert problem with the jump matrix defined by the spectral functions. We derived the global relation for the BK system involving the given initial values and the boundary values. When it comes to being well-posed, all boundary values may not be require to specify as boundary conditions. The fact that the spectral functions satisfy the global relation provides a constraint on the initial and boundary values, which makes it possible to characterize the unknown boundary values. In general, this can be done by solving nonlinear Volterra integral equations for the unknown boundary values [15]. We will analyze the global relation for the BK system in the near future.

\section{Appendix Asymptotics for eigenfunction}

In this section, we determine the asymptotic behavior of the eigenfunction $\mu$ of the Lax pair (15) as $k \rightarrow \infty$. To this end, we expand the eigenfunction as

$$
\mu(x, t, k)=I+\frac{\chi^{(1)}(x, t)}{k}+\frac{\chi^{(2)}(x, t)}{k^{2}}+\cdots, \quad(k \rightarrow \infty) .
$$


Substituting this expansion into the $x$-part of the Lax pair (15a), we find

$$
\begin{gathered}
O(1): i\left[\sigma_{3}, \chi^{(1)}\right]=U_{0}, \\
O(1 / k): \quad \chi_{x}^{(1)}+i\left[\sigma_{3}, \chi^{(2)}\right]=U_{0} \chi^{(1)}+\frac{1}{2 i} U_{1}
\end{gathered}
$$

with

$$
U_{0}=\left(\begin{array}{cc}
0 & 0 \\
-w e^{2 \int_{(0,0)}^{(x, t)} \Delta} & 0
\end{array}\right), \quad U_{1}=\left(\begin{array}{cc}
w & v e^{-2 \int_{(0,0)}^{(x, t)} \Delta} \\
0 & -w
\end{array}\right)
$$

where the closed differential one-form $\Delta(x, t)$ is defined by

$$
\Delta(x, t)=\Delta_{1} d x+\Delta_{2} d t=\frac{v}{2} d x+\frac{1}{4}(r+2 w) d t .
$$

On the other hand, substituting Eq. (57) into the $t$-part of the Lax pair (15b), we obtain

$$
\begin{gathered}
O(k):-\left[\sigma_{3}, \chi^{(1)}\right]=i V_{0}, \\
O(1):-\left[\sigma_{3}, \chi^{(2)}\right]=i V_{0} \chi^{(1)}+V_{1}, \\
O(1 / k): \chi_{t}^{(1)}-\left[\sigma_{3}, \chi^{(3)}\right]=i V_{0} \chi^{(2)}+V_{1} \chi^{(1)}+\frac{1}{4 i} V_{2},
\end{gathered}
$$

where

$$
V_{0}=\left(\begin{array}{cc}
0 & 0 \\
-w e^{2 \int_{(0,0)}^{(x, t)} \Delta} & 0
\end{array}\right), \quad V_{1}=\left(\begin{array}{cc}
0 & \frac{v}{2} e^{-2 \int_{(0,0)}^{(x, t)} \Delta} \\
-\frac{q}{2} e^{2 \int_{(0,0)}^{(x, t)} \Delta} & 0
\end{array}\right), \quad V_{2}=\left(\begin{array}{c}
q(r+w) e^{-2 \int_{(0,0)}^{(x, t)} \Delta} \\
0 \\
-q
\end{array}\right)
$$

with $r=v^{2}-v_{x}$ and $q=w_{x}+w v$.

Equations (58a) and (60a) are consistent, and we then have

$$
\chi_{12}^{(1)}=0, \quad \chi_{21}^{(1)}=\frac{w}{2 i} e^{2 \int_{(0,0)}^{(x, t)} \Delta} .
$$

Note that Eq. (58b) implies that

$$
\begin{gathered}
\chi_{12}^{(2)}=-\frac{v}{4} e^{-2 \int_{(0,0)}^{(x, t)} \Delta}, \\
\chi_{11 x}^{(1)}=\frac{w}{2 i}, \quad \chi_{22 x}^{(1)}=-\frac{w}{2 i} .
\end{gathered}
$$


From Eq. (60c), it follows that

$$
\chi_{11 t}^{(1)}=\frac{1}{4 i}(q+w v), \quad \chi_{22 t}^{(1)}=-\frac{1}{4 i}(q+w v) .
$$

Thus, letting the closed differential one-form $\Omega$ as

$$
\Omega(x, t)=w d x+\frac{1}{2}(q+w v) d t=\Omega_{1} d x+\Omega_{2} d t,
$$

the solutions to Eqs. (61b) and (62) can be found as

$$
\chi_{11}^{(1)}(x, t)=\frac{1}{2 i} \int_{(0,0)}^{(x, t)} \Omega, \quad \chi_{22}^{(1)}(x, t)=-\frac{1}{2 i} \int_{(0,0)}^{(x, t)} \Omega .
$$

Finally, we can determine the asymptotics for the eigenfunction as

$$
\mu(x, t, k)=I+\frac{1}{2 i k}\left(\begin{array}{cc}
\int_{(0,0)}^{(x, t)} \Omega & 0 \\
w e^{2 \int_{(0,0)}^{(x, t)} \Delta} & -\int_{(0,0)}^{(x, t)} \Omega
\end{array}\right)+O\left(1 / k^{2}\right), \quad(k \rightarrow \infty) .
$$

We remark that $\chi_{12}^{(2)}(x, t)$ is given by eq. (61a) and from eqs. (58b) and (60b), we find

$$
\chi_{21}^{(2)}=-\frac{q}{4} e^{2 \int_{(0,0)}^{(x, t)} \Delta}-\frac{w}{4} e^{2 \int_{(0,0)}^{(x, t)} \Delta} \int_{(0,0)}^{(x, t)} \Omega .
$$

Acknowledgements The author would like to thank anonymous reviewers, whose comments and suggestions helped improve the manuscript.

Open Access This article is distributed under the terms of the Creative Commons Attribution 4.0 International License (http://creativecommons.org/licenses/by/4.0/), which permits unrestricted use, distribution, and reproduction in any medium, provided you give appropriate credit to the original author(s) and the source, provide a link to the Creative Commons license, and indicate if changes were made.

Author Contributions The author confirms sole responsibility for study conception and design, analysis and interpretation of results, and manuscript preparation and writing.

Funding Information The author is supported by Basic Science Research Program through the National Research Foundation of Korea (NRF) funded by the Ministry of Education (NRF-2020R1F1A1A01067944).

Availability of data and material No datasets were generated or analyzed during the current study.

\section{Declarations}

Ethics approval and consent to participate Not applicable.

Consent for publication Not applicable.

Conflicts of interest The author declares no conflicts of interest. 
Open Access This article is licensed under a Creative Commons Attribution 4.0 International License, which permits use, sharing, adaptation, distribution and reproduction in any medium or format, as long as you give appropriate credit to the original author(s) and the source, provide a link to the Creative Commons licence, and indicate if changes were made. The images or other third party material in this article are included in the article's Creative Commons licence, unless indicated otherwise in a credit line to the material. If material is not included in the article's Creative Commons licence and your intended use is not permitted by statutory regulation or exceeds the permitted use, you will need to obtain permission directly from the copyright holder. To view a copy of this licence, visit http://creativecommons.org/licen ses/by/4.0/.

\section{References}

1. Broer, L.J.F.: Approximate equations for long water waves. Appl. Sci. Res. 31, 377-395 (1975)

2. Kaup, D.J.: A higher-order water-wave equation and the method for solving it. Prog. Theor. Phys. 54, 396-408 (1975)

3. Hirota, R.: Solutions of the classical Boussinesq equation and spherical Boussinesq equation: the Wronskian technique. J. Phys. Soc. Jpn. 55, 2137-2150 (1986)

4. Wang, X., Zhu, J.: Broer-Kaup system with corrections via inverse scattering transform. Avd. Math. Phys. 2020, 7859897 (2020)

5. Zhu, J., Wang, X.: Broer-Kaup system revisit: inelastic interaction and blowup solutions. J. Math. Anal. Appl. 496, 124794 (2021)

6. Ying, J.P., Lou, S.Y.: Abundant coherent structures of the (2+1)-dimensional Borer-Kaup-Kupershmidt equation. A. Naturforsch. 53, 251-258 (1998)

7. Kaup, D.J.: A perturbation expansion for the Zakharov-Shabat inverse scattering transform. SIAM J. Appl. Math. 31, 121-133 (1976)

8. Li, Y.S., Ma, W.X., Zhang, J.E.: Darboux transformations of classical Boussinesq system and its new solutions. Phys. Lett. A 275, 60-66 (2000)

9. Kupershmidt, B.A.: Mathematics of dispersive water waves. Commun. Math. Phys. 99, 51-73 (1985)

10. Satsuma, J., Kajiwara, K., Matsukidaira, J., Hietarinta, J.: Solutions of the system through its trilinear form. J. Phys. Soc. Jpn. 61, 3096-3102 (1992)

11. Meng, Q., Li, W., He, B.: Smooth and peaked solitary wave solutions of the Broer-Kaup system using the approach of dynamical system. Commun. Theor. Phys. 62, 308-314 (2014)

12. Jiang, B., Bi, Q.S.: Peaked periodic wave solutions to the Broer-Kaup equation. Commun. Theor. Phys. 67, 22-26 (2017)

13. Fokas, A.S.: A unified transform method for solving linear and certain nonlinear PDEs. Proc. Roy. Soc. Lond. A 453, 1411-1443 (1997)

14. Fokas, A. S.: A Unified approach to boundary value problems, (CBMS-NSF Regional Conference Series in Applied Mathematics) Philadelphia, SIAM (2008)

15. Fokas, A.S.: Integrable nonlinear evolution equations on the half-line. Commun. Math. Phys. 230, 1-39 (2002)

16. Lenells, J.: The derivative nonlinear Schrödinger equation on the half-line. Phys. D 237, 3008-3019 (2008)

17. Fokas, A.S.: A new transform method for evolution partial differential equations. IMA J. Appl. Math. 67, 559-590 (2002)

18. Deconinck, B., Trogdon, T., Vasan, V.: The method of Fokas for solving linear partial differential equations. SIAM Rev. 56, 159-186 (2014)

19. Pelloni, B., Pinotsis, D.A.: The elliptic sine-Gordon equation in a half plane. Nonlinearity $\mathbf{2 3}, \mathbf{7 7 - 8 8}$ (2010)

20. Colbrook, M.J., Flyer, N., Fornberg, B.: On the Fokas method for the solution of elliptic problems in both convex and non-convex polygonal domains. J. Comput. Phys. 374, 996-1016 (2018)

21. Hwang, G.: The elliptic sinh-Gordon equation in a semi-strip. Adv. Nonlinear Anal. 8, 533-544 (2019) 
22. Biondini, G., Hwang, G.: Initial-boundary value problems for discrete evolution equations: discrete linear Schrödinger and integrable discrete nonlinear Schrödinger equations. Inv. Probl. 24(065011), 1-44 (2008)

23. Moon, B., Hwang, G.: Discrete linear evolution equations in a finite lattice. J. Differ. Equ. Appl. 25, 630-646 (2019)

24. Tian, S.F.: The mixed coupled nonlinear Schrodinger equation on the half-line via the Fokas method. Proc. R. Soc. A 472, 20160588 (2016)

25. Tian, S.F.: Initial-boundary value problems for the general coupled nonlinear Schrödinger equation on the interval via the Fokas method. J. Differ. Equ. 262, 506-558 (2017)

26. Tian, S.F.: Initial-boundary value problems for the coupled modified Korteweg-de Vries equation on the interval. Commun. Pure Appl. Anal. 17, 923-957 (2018)

27. Fokas, A.S.: The generalized Dirichlet-to-Neumann map for certain nonlinear evolution PDEs. Comm. Pure Appl. Math. LVII I, 639-670 (2005)

28. Fokas, A.S., Pelloni, B.: The Dirichlet-to-Neumann map for the elliptic sine-Gordon equation. Nonlinearity 25, 1011-1031 (2012)

29. Hwang, G.: The Fokas method: the Dirichlet to Neumann map for the sine-Gordon equation. Stud. Appl. Math. 132, 381-406 (2014)

30. Crowdy, D.G., Luca, E.: Solving Wiener-Hopf problems without kernel factorization. Proc. R. Soc. A 470, 20140304 (2014)

31. Colbrook, M.J., Ayton, L.J., Fokas, A.S.: The unified transform for mixed boundary condition problems in unbounded domains. Proc. R. Soc. A 475, 20180605 (2019)

32. Deift, P., Zhou, X.: A steepest descent method for oscillatory Riemann-Hilbert problems. Bull. Am. Math. Soc. 26, 119-123 (1992)

33. Deift, P., Venakides, S., Zhou, X.: New results in small dispersion KdV by an extension of the steepest descent method for Riemann-Hilbert problems. Int. Math. Res. Not. 6, 286-299 (1997)

34. Lenells, J., Fokas, A.S.: The unified method on the half-line: II. NLS on the half-line with $t$-periodic boundary conditions. J. Phys. A Math. Theor. 45, 195202 (2012)

35. Hwang, G., Fokas, A.S.: The modified Korteweg-de Vries equation on the half-line with a sinewave as Dirichlet datum. J. Nonlinear Math. Phys. 20, 135-157 (2013)

36. Lenells, J., Fokas, A.S.: The nonlinear Schrödinger equation with $t$-periodic data: II. Perturbative results. Proc. R. Soc. A 471, 20140926 (2015)

37. Hwang, G.: A perturbative approach for the asymptotic evaluation of the Neumann value corresponding to the Dirichlet datum of a single periodic exponential for the NLS. J. Nonlinear Math. Phys. 21, 225-247 (2014)

38. Hwang, G.: The modified Korteweg-de Vries equation on the quarter plane with $t$-periodic data. J. Nonlinear Math. Phys. 24, 620-634 (2017)

39. Colbrook, M.J., Fokas, A.S., Hashemzadeh, P.: A hybrid analytical-numerical technique for elliptic PDEs. SIAM J. Sci. Comput. 41, A1066-A1090 (2019)

40. de Barros, F.R.J., Colbrook, M.J., Fokas, A.S.: A hybrid analytical-numerical method for solving advection-dispersion problems on a half -line. Int. J. Heat Mass Transf. 139, 482-491 (2019)

41. Hwang, G.: Initial-boundary value problems for the one-dimensional linear advection-dispersion equation with decay. Z. Naturforshc. A 75, 713-725 (2020) 